\title{
Neutron Generation and Diffusion Process in Accelerator-Driven System Reactors
}

\author{
B.M. Santos $^{a *}$, R.G. Cabral ${ }^{b}$, M. Gonçalves ${ }^{c}$ and S.B. Duarte ${ }^{d}$ \\ ${ }^{a}$ Instituto de Física, Universidade Federal Fluminense \\ Av. Gal. Milton Tavares de Souza, 24210-346 Niterói-RJ, Brasil \\ ${ }^{b}$ Instituto Militar de Engenharia \\ Praça Gal. Tibúrcio 80, 22290-270 Rio de Janeiro-RJ, Brasil \\ ${ }^{c}$ Comissão Nacional de Energia Nuclear \\ Rua General Severiano 90, 22290-901 Rio de Janeiro-RJ, Brasil \\ ${ }^{d}$ Centro Brasileiro de Pesquisas Físicas \\ Rua Dr. Xavier Sigaud 150, 22290-180 Rio de Janeiro-RJ, Brasil \\ E-mail: biank_ce@if.uff.br
}

\begin{abstract}
This work introduces a new treatment for the spallation reaction, which is the main mechanism for neutron generation in ADS reactors. These reactions are conventionally described by two coupled stages with different scales in time. First a rapid intranuclear cascade, followed by a slow particle evaporation process in competition with the fission process. The cascade phase was described by a time-dependent Multi-Collisional Monte Carlo (MCMC) numerical code, where many-particle system evolves in time along the cascade. This work introduces, in the cascade phase, a baryon effective mass, to better represent the effect of nuclear binding energy on the nucleon kinematics. We discuss the implications of the inclusion of this baryon effective mass to the evaporation phase, and to the particles production at the end of the whole reaction process. The diffusion of neutrons was analytically solved by improving the treatment presented in a recent work [1], by using two groups of energy. It is exhibited an analytical solution even using this more precise approximation. We explicitly show the dependence of the rector power on the neutron source current, and determines the power for different levels of subcritical operational regime of the reactor.
\end{abstract}

XXXIV edition of the Brazilian Workshop on Nuclear Physics,

5-10 June 2011

Foz de Iguaçu, Paraná, Brazil

\footnotetext{
* Speaker.
} 


\section{Introduction}

The Accelerator-Driven System (ADS) is an innovative reactor with the aim of burning long radioactive half-life waste. This device was proposed by Rubbia et al. [2], and is essentially composed by a particle accelerator around $1 \mathrm{GeV}$ nominal energy, which induces spallation reactions in liquid lead target. This reaction generates neutrons to form the external source for a sub-critical reactor, where the liquid lead works as the reactor coolant itself, circulating by natural convection.

Spallation reactions induced by protons have been well described as a two-step process. In the first step, the incident proton transfers energy to the target nucleus, inducing an intranuclear cascade process. At this stage, individual nucleons can be ejected, and mesons can be produced. Particles escape from the nucleus carrying out part of the energy transferred by the incident proton. The remaining energy is redistributed by elastic collisions leading to thermal equilibrium of a hot excited nucleus. The subsequent step is the particle evaporation phase, where the cascade residual nucleus de-excites by emitting particles in competition with the fission process. This last phase is the most relevant to the production of neutrons. However, it is the cascade phase that determines the excitation energy of the residual nucleus, the most relevant quantity to the development of the evaporation phase. One of the main goals of this work is to investigate the role of the nucleon effective mass to the excitation energy of the cascade residual nucleus.

In addition, we describe the neutron diffusion process in order to elucidate the effect of the neutron source current on the reactor power for different levels of the reactor sub-criticality. We decided to look for analytic solutions of the neutron diffusion equation, applying the two-energy group diffusion treatment as an improvement to the recent calculation presented in Ref. [1]. The use of an analytic approach has the main advantage of making transparent the influence of the neutron source intensity on the study of the reactor sub-criticality level.

\section{Spallation reaction description and cascade phase}

The spallation reaction is the mechanism responsible for the neutron generation in ADS reactors. We have implemented a Monte Carlo simulation of the reaction process, including two stages: the intranuclear cascade phase and the particle evaporation phase in competition with the fission process. The Multicollisional Monte Carlo (MCMC) code is used to perform the computational simulation $[3,4]$.

In the intranuclear cascade, the proton beam interacts with a nucleon of the target nucleus as the primary collision process. Then, successive uncorrelated binary nucleon-nucleon collisions continue along the time. The time step is defined as the inter-collision time interval. After each nucleon-nucleon collision, all $(A+1)$-nucleons in the system have their spatial coordinates updated to re-calculate the next collision to be processed. The starting point for simulation consists in specifying the initial nuclear configuration of nucleons inside the target nucleus. The nuclear surface is simulated by a sphere of radius $R=r_{0} A^{1 / 3}$ (with $r_{0}=1.18 \mathrm{fm}$ ), where $A$ is the mass number of the target nucleus, and initial position for nucleons are randomly generated via Monte Carlo, according to a homogeneous density distribution [4-6]. The total baryon-baryon cross section is extracted from experimental data presented in the literature [7-10]. Binary collisions are solved kinematically and the final state of the pair of particles is determined by relativistic energy-momentum 
conservation. Baryon-baryon inelastic collisions are also considered; thus pions, kaons and heavy baryonic resonances can be generated along the system time evolution. Micro-processes regarded in the calculation are shown in Table 1.

Table 1: Collisional processes considered in the MCMC description.

\begin{tabular}{lc}
\hline \hline Interaction & Description \\
\hline$B_{1} B_{2} \rightarrow B_{1} B_{2}$ & Baryon-baryon elastic collision \\
$N N \rightleftharpoons N \Delta$ & Formation and recombination of the $\Delta$-resonance \\
$N N \rightleftharpoons N N^{*}$ & Formation and recombination of the $N^{*}$-resonance \\
$\Delta \rightleftharpoons N \pi$ & $\Delta$-resonance decay and pion absorption \\
$N^{*} \rightleftharpoons N \pi$ & $N^{*}$-resonance decay and pion absorption \\
\hline \hline
\end{tabular}

The Pauli Exclusion Principle was considered by examining the availability of the phase space to the final state of the particles involved in the collision [11].

The cascade phase ends when the particle production and the particle emission processes cease. However, it is still necessary to achieve the relaxation of the particles energy distribution, when a thermal equilibrium is reached by the residual nucleus configuration. Then the evaporation regime starts with excitation energy $E^{*}$ defined at the end of the cascade phase. A competition between the chain of thermal emission of particles (protons, neutrons and alpha particles) and fission process is initiated, being the whole evaporation regime governed by relative probabilities of different channels [12-21].

\subsection{The evaporation phase}

In this phase, a chain of thermal emission of particles is initiated in competition with the fission process. The ratio between the decay width of $k$-th and of $i$-th emission channels is given by the Weisskopf's statistical model [12], as

$$
\frac{\Gamma_{k}}{\Gamma_{i}}=\frac{\gamma_{k}}{\gamma_{i}} \frac{E_{k}^{*}}{E_{i}^{*}} \frac{a_{i}}{a_{k}} \exp \left[2\left(\sqrt{a_{k} E_{k}^{*}}-\sqrt{a_{i} E_{i}^{*}}\right)\right] .
$$

The level density parameter for neutron emission is [13]

$$
a_{n}=\widetilde{a}\left\{1+\left[1-\exp \left(-0.051 E^{*}\right)\right] \frac{\Delta M}{E^{*}}\right\},
$$

where $\Delta M$ (expressed in $\mathrm{MeV}$ ) is the shell correction in the calculated nuclear mass taken from [14], and

$$
\widetilde{a}=0.114 A+0.098 A^{2 / 3} \mathrm{MeV}^{-1} .
$$

Small corrections on $E^{*}$ due to pairing energy effects have been neglected in equation (2.2). For all other particle emission processes this quantity is related to $a_{n}$ by

$$
a_{k}=r_{k} a_{n}
$$

where $r_{k}$ is a dimensionless constant. For the $k$-th particle, $\gamma_{k}=g_{k} m_{k} /\left(\pi^{2} h^{3}\right)$, where $h$ is the Planck constant, $g_{k}$ is the number of spin states ( $g_{k}=2$ for neutrons and protons; $g_{k}=1$ for $\alpha$-particle) and $m_{k}$ is the particle free rest mass. 
The excitation energy of the nucleus after the $k$-particle emission is

$$
E^{*}=E^{*}-\bar{E}_{k}^{e v}
$$

where

$$
\bar{E}_{k}^{e v}=\left(S_{k}+V_{k}\right)+2 \sqrt{\frac{E^{*}-\left(S_{k}+V_{k}\right)}{a_{n}}}
$$

is the average total energy removed from the nuclear system by the evaporation of $k$-particle according to a Weisskopf distribution curve [15], for $k=n, p$ or $\alpha$ and $V_{n}=0$. The approximation $a_{n}=a_{p}=a_{\alpha}\left(r_{p}=r_{\alpha}=1\right.$ in Eq. (2.3), as prescribed in Ref. [18]) was considered and $S_{n}, S_{p}$ and $S_{\alpha}$ are the separation energies for neutron, proton and alpha particle, respectively.

The Coulomb potential for protons is $[16,17]$

$$
V_{p}=C \frac{K_{p}(Z-1) e^{2}}{r_{0}(A-1)^{1 / 3}+R_{p}}
$$

and for alpha particle is

$$
V_{\alpha}=C \frac{2 K_{\alpha}(Z-2) e^{2}}{r_{0}(A-4)^{1 / 3}+R_{\alpha}}
$$

where $K_{p}=0.70$ and $K_{\alpha}=0.83$ are the Coulomb barrier penetrability for protons and alpha particles, respectively, $R_{p}=1.14 \mathrm{fm}$ is the proton radius, $R_{\alpha}=2.16 \mathrm{fm}$ is the alpha particle radius, $r_{0}=1.2 \mathrm{fm}$, and

$$
C=1-\frac{E^{*}}{B}
$$

is the charged-particle Coulomb barrier correction due to nuclear temperature ( $B$ as the total nuclear binding energy).

The relative fission probability (see ref. [21]) is obtained using the fission width from the liquid drop model by Bohr and Wheeler [20], and the neutron emission width from Weisskopf theory [12]. The MCMC code determines, for each step of the evaporation phase, the nuclear fission, neutron, proton, and alpha particle emission probabilities ( $F, N, P$ and $A$, respectively) by

$$
\begin{aligned}
& F=\frac{\Gamma_{f}}{\Gamma_{n}+\Gamma_{p}+\Gamma_{\alpha}+\Gamma_{f}} ; \\
& N=\frac{\Gamma_{n}}{\Gamma_{n}+\Gamma_{p}+\Gamma_{\alpha}+\Gamma_{f}} ; \\
& P=\frac{\Gamma_{p}}{\Gamma_{n}+\Gamma_{p}+\Gamma_{\alpha}+\Gamma_{f}} ; \\
& A=\frac{\Gamma_{\alpha}}{\Gamma_{n}+\Gamma_{p}+\Gamma_{\alpha}+\Gamma_{f}}
\end{aligned}
$$

Finally, the evaporation chain is governed by relative branching ratios of Eqs. (2.6-2.9) via Monte Carlo technique. 


\subsection{The role of nucleon effective mass to the cascade phase}

The initial effective mass of bound nucleons, $m^{*}$, is taken as a free parameter, covering the typical values obtained by mean-field calculation $[22,23]$ to finite nuclei. During the time evolution of the system, particles leaving the nuclear environment have effective mass value restored to the free nucleon mass. To preserve the total energy and momentum conservation of the system, the remaining bound nucleons must have their effective mass also changed as $\left(m_{i}^{*} \rightarrow \alpha m_{i}^{*}\right)$, with the factor $\alpha$ determined by,

$$
\alpha=\frac{E\left(E+\varepsilon_{i}\right)+\sqrt{\left[E\left(E+\varepsilon_{i}\right)\right]^{2}-\left[E^{2}-p_{i}^{2}\right]\left[\left(E+\varepsilon_{i}\right)^{2}-m_{0}^{2}\right]}}{E^{2}-p_{i}^{2}},
$$

where $m_{0}$ is the baryon free mass, $\varepsilon_{i}=\sqrt{p_{i}^{2}+m_{i}^{* 2}}$ is the energy of the $i$ particle, $p_{i}$ is the corresponding momentum and $E=\left(\Sigma_{j} \varepsilon_{j}\right)$, being the subscript $i$ used for emitted particle and the subscript $j$ to bound particles. In Fig. 1, the time evolution of bound nucleons effective mass during the cascade phase, starting with different initial values, is shown. The reaction is $p+{ }^{208} \mathrm{~Pb}$ at 1 $\mathrm{GeV}$ incident energy.

The use of an effective mass varying in time implies different kinematic relations for successive intranuclear collisions (see Fig. 1). It affects directly the energy transference leading to different final excitation energy results for the cascade residual nucleus, as shown in Fig. 2.

The excitation energy and number of emitted particles are determined at the end of the rapid and pre-equilibrium phase. These are the key values to start the evaporation phase. Consequently, changes in the baryonic effective mass during cascade phase implies different evaporative chains from the same hot nucleus. The emitted particle yield and the energy available for fission events are directly dependent on the residual nucleus excitation energy. In Table 2, results of the neutron yield of rapid and slow phases are presented. One can clearly observe the dominance of the evaporation phase for the yield of neutrons in the whole reaction process.

Table 2: Average multiplicity of neutron, proton, pions and alpha ( $n, p, \pi$ and $\alpha$, respectively) produced in the cascade and evaporation phases. Different initial values of the effective mass, $m^{*}$ (in units of the nucleon free mass, $\left.m_{0}\right)$ were adopted. The average number of fission $(f)$ of the residual hot nucleus in the evaporation phase is also shown. The beam energy is $1 \mathrm{GeV}$ incident on a Pb-208 target. The cascade average residual nucleus excitation energy, $E^{*}$, is also presented.

\begin{tabular}{c|ccc|ccc|ccc|ccc}
\hline \hline & \multicolumn{3}{|c|}{$m^{*}=0.6 m_{0}$} & \multicolumn{3}{c|}{$m^{*}=0.7 m_{0}$} & \multicolumn{3}{c|}{$m^{*}=0.8 m_{0}$} & \multicolumn{3}{c}{$m^{*}=0.9 m_{0}$} \\
& \multicolumn{2}{|c|}{$\left(E^{*}=174.1 \mathrm{MeV}\right)$} & \multicolumn{2}{c}{$\left(E^{*}=189.9 \mathrm{MeV}\right)$} & \multicolumn{2}{c}{$\left(E^{*}=209.6 \mathrm{MeV}\right)$} & \multicolumn{2}{c}{$\left(E^{*}=238.9 \mathrm{MeV}\right)$} \\
\hline & Casc & Evap & Total & Casc & Evap & Total & Casc & Evap & Total & Casc & Evap & Total \\
\hline$n$ & 0.73 & 7.04 & 7.76 & 0.74 & 8.17 & 8.92 & 0.75 & 8.36 & 9.11 & 0.70 & 7.76 & 8.46 \\
$p$ & 1.36 & 0.08 & 1.44 & 1.35 & 0.10 & 1.45 & 1.32 & 0.07 & 1.39 & 1.23 & 0.09 & 1.32 \\
$\pi$ & 0.12 & - & 0.12 & 0.13 & - & 0.13 & 0.15 & - & 0.15 & 0.15 & - & 0.15 \\
$\alpha$ & - & 0.17 & 0.17 & - & 0.18 & 0.18 & - & 0.08 & 0.08 & - & 0.08 & 0.08 \\
$f$ & - & 0.88 & 0.88 & - & 0.88 & 0.88 & - & 0.84 & 0.84 & - & 0.80 & 0.80 \\
\hline \hline
\end{tabular}

Another important result (not shown) is the calculated particle spectra. We observe that cascade phase produces high energy neutrons $\left(T_{n}>100 \mathrm{MeV}\right)$, whereas the neutrons with moderated energies $\left(T_{n} \approx 8 \mathrm{MeV}\right)$ are originated from the evaporation phase. 


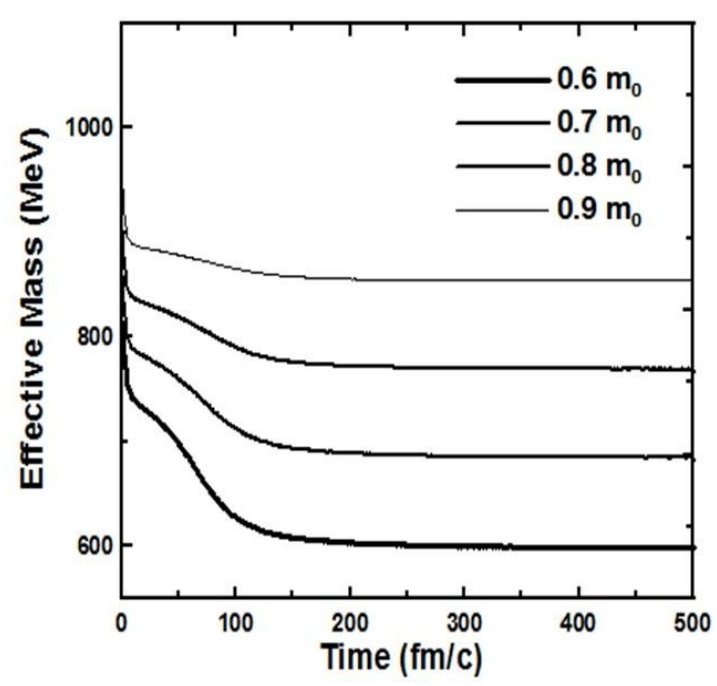

Figure 1: Time evolution of the effective mass along the cascade phase, showing the decreasing in the effective mass due to particle emission.

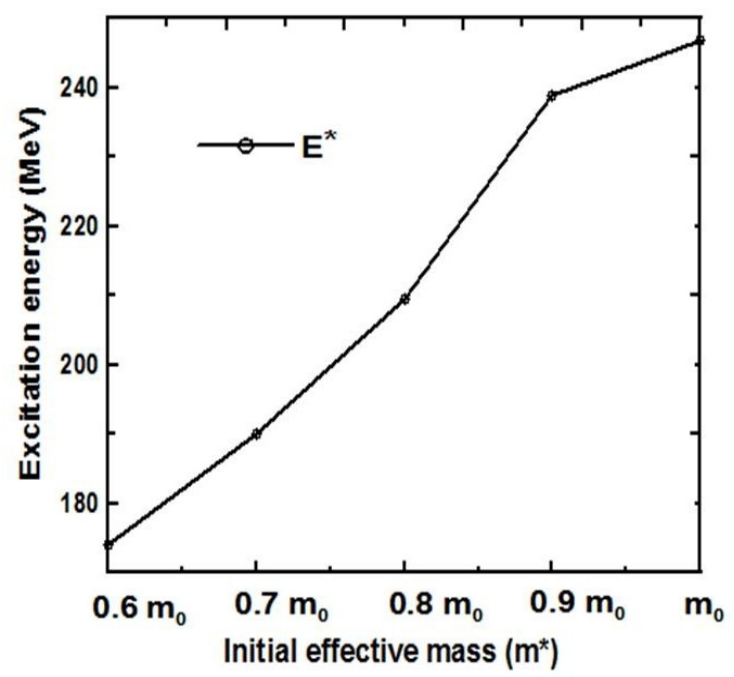

Figure 2: Relation between the excitation energy of the cascade residual nucleus and the variation of nucleons effective mass.

\section{Neutron diffusion for spherical reactor core}

The neutron diffusion process in the reactor core has been well described by the neutron transport equation [24]. Using the analytic description for two-energy groups approximation, an extension of the calculation presented in Ref. [1], one can treat the diffusion problem in terms of the following equations:

$$
-D_{1} \nabla^{2} \Phi_{1}+\Sigma_{R 1} \Phi=\frac{1}{k_{\mathrm{eff}}}\left(v_{1} \Sigma_{f 1} \Phi_{1}+v_{2} \Sigma_{f 2} \Phi_{2}\right)
$$


and

$$
-D_{2} \nabla^{2} \Phi_{2}+\Sigma_{a 2} \Phi_{2}=\Sigma_{S 12} \Phi_{1}
$$

for $(0<r \leq R)$, where $D_{1}$ and $D_{2}$ are the diffusion coefficients of neutron of group-1 and group2, respectively; $\Phi_{1}=\Phi_{1}(r)$ and $\Phi_{2}=\Phi_{2}(r)$ are the corresponding neutron flux; $\Sigma_{R 1}=\Sigma_{S 12}+$ $\Sigma_{a 1}$ is the macroscopic cross section of neutron removal, $\Sigma_{S 12}$ is the macroscopic cross section of scattering from group-1 to group-2; $\Sigma_{a 1}$ and $\Sigma_{a 2}$ are the cross sections of neutron absorption process for the corresponding neutron groups; $\Sigma_{f 1}$ and $\Sigma_{f 2}$ are the cross sections of induced fission by neutrons of groups 1 and 2; $v_{1}$ and $v_{2}$ are the average number of produced neutrons in fission induced by group- 1 and group- 2 neutrons.

In Eqs. 3.1 and 3.2 the criticality of the diffusion process is controlled by the $k_{\text {eff }}$ parameter defined as,

$$
k_{\mathrm{eff}}=\frac{v_{1} \Sigma_{f 1}+\left(v_{2} \Sigma_{f 2} \Sigma_{S 12}\right) /\left(\Sigma_{a 2}+D_{2} \mu^{2}\right)}{\Sigma_{R 1}+D_{1} \pi^{2} / R^{2}}
$$

This system of equations satisfies the following boundary conditions:

$$
\begin{array}{rlrl}
\text { (i) } \Phi_{1}(r=\widetilde{R}) & =0 ; & & \text { (iii) } r=0 \rightarrow 4 \pi r^{2} \vec{J}_{1}=I_{0} \vec{a}_{r} ; \\
\text { (ii) } \Phi_{2}(r=\widetilde{R})=0 ; & & \text { (iv) } r=0 \rightarrow 4 \pi r^{2} \vec{J}_{2}=0 ;
\end{array}
$$

with $I_{0}$ being the source flux. This flux is directly determined by the results of spallation reaction mechanism as discussed in previous section.

The solution of the simultaneous linear differential equation (3.1 and 3.2) reads:

$$
\Phi_{i}=\frac{I_{0}}{r}\left(c_{1 i} \sin \mu r+c_{2 i} \cos \mu r+c_{3 i} \sinh \lambda r+c_{4 i} \cosh \lambda r\right),
$$

in which the constants are obtained by the use of the boundary conditions in Eqs. 3.4 and 3.5 (for $i=1,2$ according to the energy group).

The reactor power was determined as,

$$
P=I_{0} \int_{r=0}^{R} \Sigma_{f}\left[W_{1} \varphi_{1}(r)+W_{2} \varphi_{2}(r)\right] 4 \pi r^{2} d r
$$

where $\varphi_{i}$ is the flux per source current intensity (see Fig. 3), and $W_{1}$ and $W_{2}$ are the effective energy released in fast and thermal fission processes.

Neutron fluency can be determined by neutron multiplicities studied in the previous section, and by using constants of a typical PWR ("Pressurizer Water Reactor") sub-critical neutron flux [24], which is shown in Fig. 3. It was observed that the reactor power is proportional to the intensity of the source (as shown in Eq. 3.7). The response of the reactor in the sub-critical regime is more significant for higher values of $k_{\text {eff }}$ as shown in Fig. 4. 


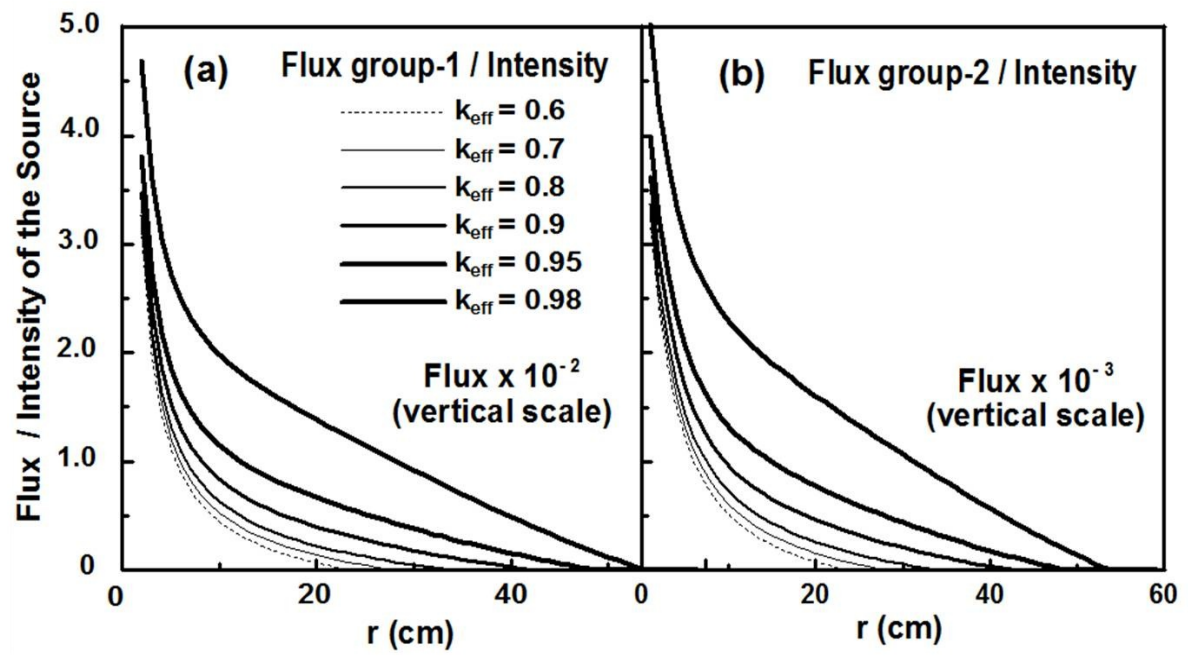

Figure 3: Neutron flux of group-1 per source intensity as a function of distance to the source in part-(a). Same result for group-2 neutron in part-(b). For different values of the effective factor of neutrons, $k_{\text {eff }}=$ $0.6,0.7,0.8,0.9,0.95$ and 0.98 .

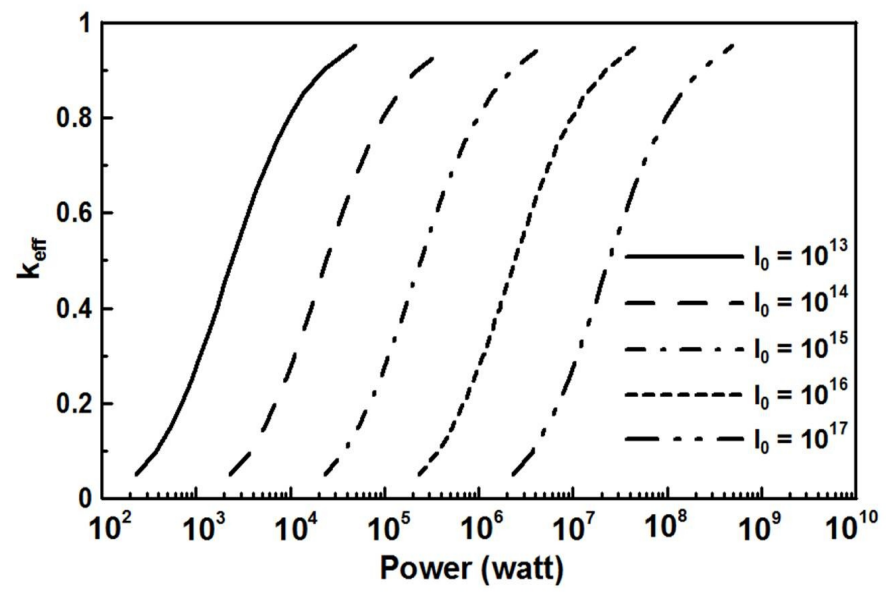

Figure 4: Effect of the source current intensity $I_{0}$ (in neutrons/sec) on reactor power for different subcriticality levels.

\section{Conclusions}

In the study of the neutron source, we determined the importance of baryonic effective mass to the particle yield, specially to the neutron multiplicity, which is a key element to calculate the source current for the ADS nuclear reactor device. We have investigated the excitation energy of the hot residual cascade nucleus. Results point out to a significant dependence of this excitation energy on the effective mass of nucleons in cascade phase.

In the neutronic calculation of the sub-critical reactor, we have used a two-energy groups approach to solve the neutron diffusion equation. This treatment allows to establish analytically a direct relationship between power generation and neutron source current (Eq. 3.7), and also permits 
to easily analyze the reactor power for different levels of the reactor sub-criticality, as shown in Fig. 4.

\section{References}

[1] P. Saracco, N. Science Eng., 162 (2009) 167.

[2] C. Rubbia et al., European Organization for Nuclear Research Report, (1995) [CERN/AT/95-44(ET) ].

[3] M. Gonçalves et al., Phys. Lett. B 406 (1997) 1.

[4] M. Gonçalves, E.L. Medeiros and S.B. Duarte, Phys. Rev. C, 55 (1996) 5.

[5] T. Kodama, S.D. Duarte, K.C. Chung and R.A.M.S. Nazereth, Phys. Rev. Letters, 49 (1982) 8.

[6] T. Kodama, S.D. Duarte, K.C. Chung, R. Donangelo and R.A.M.S. Nazereth, Phys. Rev. C, 29 (1983) 6.

[7] Particle Data Group, NN and ND Interactions, G.J. Igo, Rev. Mod. Phys. 50 (1978) 523.

[8] Y. Kitazoe, M. Sano, Y. Yamamura, H. Furutani and K. Yamamoto, Phys. Rev. C 29 (1984) 828.

[9] E.L. Medeiros, S.B. Duarte and T. Kodama, Phys. Lett. B, 203 (1988) 205.

[10] B.J. VerWest and R.A. Arndt, Phys. Rev. C, 25 (1982) 4.

[11] S.R. Pina, J. Mesa, A. Deppman, J. D. T. Arruda-Neto, S.B. Duarte, O. A. P. Tavares, E. C. Oliveira; E. L. Medeiros, M.G. Gonçalves, E. Paiva, Phys. G: Nucl. Part. Phys.,28 (2002) 2259.

[12] V.F. Weisskopf, Phys. Rev. 52 (1937) 295.

[13] O.A.P. Tavares, E.L. Medeiros, J. Phys. G: Nucl. Part. Phys.,30 (2004) 395.

[14] W.D. Myers, Droplet Model of Atomic Nuclei, 1st ed., New York: Plenum, (1977).

[15] O.A.P. Tavares et al, J. Phys. G: Nucl. Part. Phys., 30 (2004) 377.

[16] A. Deppman et al, Comp. Phys. Comm., 145 (2002) 385.

[17] A. Deppman et al, Nucl. Inst. and Meth. in Phys. Res. B 211 (2003) 15.

[18] K.J. LeCouteur, Proc. Phys. Soc. Lond., Ser. A 63 (1950) 259.

[19] C. Guaraldo et at., Nuovo Cimento 103A (1990) 607.

[20] N. Bohr, J.A. Wheeler, Phys. Rev. , 56 (1939) 426

[21] R. Vandenbosch, J.R. Huizenga, Nuclear Fission, 1 ed., Academic Press, New York (1973) p. 227.

[22] B.D. Serot and J.D. Walecka, Adv. Nucl. Phys. 16 (1986) 1.

[23] M. Bender and P.H. Heenen, Reviews of Modern Physics 75 (2003).

[24] S. D Duderstadt, Multigroup Diffusion Theory, in Nuclear reactor analysis, edited by John Wiley \& Sons Inc., New York (1976) p. 285-314. 